\title{
FAR-INFRARED REFLECTIVITY AND DYNAMICAL CONDUCTIVITY OF $\mathrm{YBa}_{2} \mathrm{Cu}_{3} \mathrm{O}_{7}$
}

\author{
W. OSE, P.E. OBERMAYER, H.H. OTTO, T. ZETTERER, H. LENGFELLNER, N. TASLER, J. KELLER and \\ K.F. RENK
}

Institut für Angewandte Physik, Universität Regensburg, 8400 Regensburg, F.R.G.

For a sintered $\mathrm{YBa}_{2} \mathrm{Cu}_{3} \mathrm{O}_{7}$ sample that shows high far-infrared reflectivity we determined, by a Kramers-Kronig analysis, the dynamical conductivity and extracted contributions by free charge carriers and phonons. The low temperature conductivity, and also results of a tunnel experiment, can be described by a distribution of energy gaps around $2 \Delta / k T_{c} \simeq 4.6$. Furthermore we present evidence for superconducting fluctuations and we show that the lowest-frequency infrared-active phonon mode is less damped in the superconducting state than in the normal state.

\section{INTRODUCTION}

From far-infrared reflection studies of $\mathrm{YBa}_{2} \mathrm{Cu}_{3} \mathrm{O}_{7}$ ceramic samples energy gaps $2 \Delta / k T_{c} \simeq 2.2$ to $2.5(1-4)$ and from a study of a single crystal sample a value of 8 (5) have been suggested. In this paper we report reflectivity measurements, and also a tunnel experiment, on a ceramic sample from which we derive a value of about 4.6. Superconducting fluctuations and properties of infrared-active phonon modes are discussed, too.

\section{SAMPLE CHARACTERIZATION}

We have prepared $\mathrm{YBa}_{2} \mathrm{Cu}_{3} \mathrm{O}_{7}$ ceramic pellets from highly pure powders. Though the treatment was very similar for various samples, one of them was highly reflecting in the far-infrared. This sample, discussed in this paper, shows a large Meissner effect and has a resistivity of about $300 \mu \Omega \mathrm{cm}$ at $300 \mathrm{~K}$ and $100 \mu \Omega \mathrm{cm}$ at $100 \mathrm{~K}$. The superconducting transition is sharp $\left(T_{c} \simeq 86 \mathrm{~K}\right)$. X-ray studies showed that the sample consists of single phase material and that the crystallites have a preferential orientation with the ab-plane in the pellet plane. According to a micrograph the surface has parket-like structure with crystallites of a typical size of $10 \mu \mathrm{m} \times 2 \mu \mathrm{m}$; the surface roughness is few $\mu m$. The density is $87 \%$ of the X-ray density $\left(6.39 \mathrm{~g} / \mathrm{cm}^{3}\right)$. A more detailed description of the sample is given elsewhere (6).

\section{FAR-INFRARED REFLECTIVITY}

The reflectivity (Fig. 1) is high at small frequencies and decreases towards large frequencies and shows pronounced phonon structure. For $T<<T_{c}$ the reflectivity (dashed) is constant and almost 1 at small frequencies and decreases, at $380 \mathrm{~cm}^{-1}$, below the normal state reflectivity (for 110 $\mathrm{K}$ ). According to a strong cut-off in the reflectivity curve we may suggest an energy gap frequency with a value of $285 \mathrm{~cm}^{-1}$ or $2 \Delta / k T_{c} \simeq 4.6$. An analysis of our results (chapter 4) supports this choice.

While the reflection was almost specular in the range shown in Fig. 1, it became mainly diffuse at larger frequencies (6). We note that reflectivity curves published hitherto for $\mathrm{Y}-\mathrm{Ba}-\mathrm{Cu}-\mathrm{O}$ (and also $\mathrm{La}-\mathrm{Ba}-\mathrm{Cu}-\mathrm{O}$ ) ceramic samples (1-4) lie below our curves, especially for low temperature. This shows that our sample has a high quality

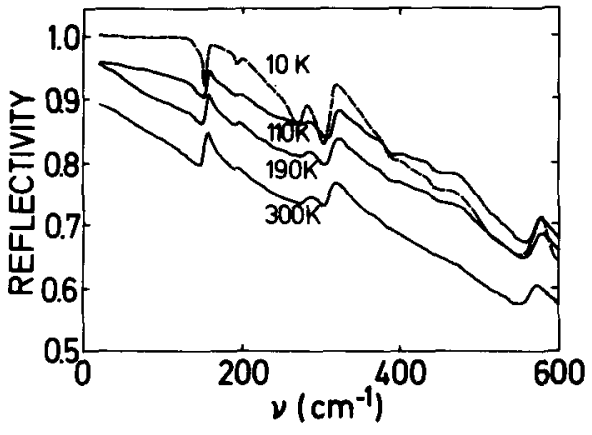

Fig. 1 Far-infrared reflectivity of a $\mathrm{YBa}_{2} \mathrm{Cu}_{3} \mathrm{O}_{7}$ ceramic sample.

in the surface region and, as a consequence, we find a higher gap value than concluded from earlier far-infrared measurements.

\section{DYNAMICAL CONDUCTIVITY}

We have determined the dynamical conductivity $\sigma=$ $\sigma_{1}+i \sigma_{2}$ by a Kramers-Kronig analysis; for the analysis we extrapolated the reflection data towards small and large frequencies taking account of diffuse reflection and by assuming smooth behavior (6). We find that $\sigma_{1}$ is not a constant for fixed temperature $\left(T>T_{c}\right)$ as expected from Drude's theory, but shows an increase towards small frequencies (left part of Fig. 2). The increase is strongest near $T_{c}$. We attribute this effect to superconducting fluctuations. At low temperature $\sigma_{1}$ is almost zero at small frequencies as expected for a superconductor with an energy gap. While a Drude behavior would lead to small values of $\sigma_{2}$ in the frequency range of our experiment and for $T>T_{c}$, we find a strong contribution that increases strongly for $T \rightarrow T_{c}$ (right part of Fig. 2). For $T=110 \mathrm{~K}$, $\sigma_{2}$ has a maximum near $60 \mathrm{~cm}^{-1}$. For $T<<T_{c} \sigma_{2}$ increases continuously towards small frequencies. The dynamical conductivity curves are mainly determined by the free carrier conductivities $\sigma_{1}^{c}$ and $\sigma_{2}^{c}$ (dashed in Fig. 2) while the fine structure is due to phonons. 


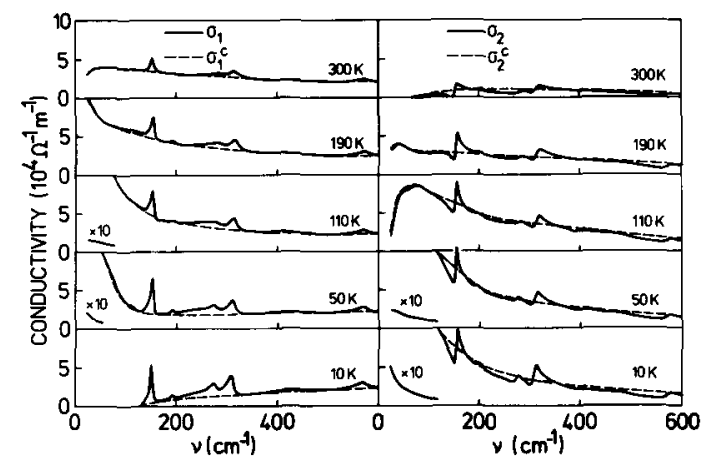

Fig. 2 Dynamical conductivities of a $\mathrm{YBa}_{2} \mathrm{Cu}_{3} \mathrm{O}_{7}$ ceramic sample.

We find five infrared active phonon modes. Three (at $567,312,278 \mathrm{~cm}^{-1}$ ) are slightly softer in the superconducting state than in the normal state and their oscillator strengths increase towards smaller frequencies and their halfwidths are independent of temperature. Another mode (at $192 \mathrm{~cm}^{-1}$ ) shows only an increase of oscillator strength with decreasing temperature. For the lowest-frequency mode $\left(\right.$ at $153 \mathrm{~cm}^{-1}$ ) both the oscillator strength and the halfwidth decrease by a factor of two. This indicates that this mode has a direct interaction with the charge carriers responsible for superconductivity. The vibration corresponds to a $\mathrm{Ba}-\mathrm{O}$ vibration near the $\mathrm{Cu}-\mathrm{O} a-b$ planes. Therefore, our result is consistent with superconductivity in these planes.

The temperature as well as the frequency dependence of the conductivities for $T>T_{c}$ are consistent with twodimensional fluctuation in conducting planes of a characteristic thickness of several Angströms (6).

For a discussion of the properties at low temperature we introduce the dynamical resistivity $\rho_{1}+i \rho_{2}=\left(\sigma_{1}^{c}+i \sigma_{2}^{c}\right)^{-1}$. We find (Fig. 3 ) that the experimental curves can qualitatively be described by the theory of Mattis und Bardeen (7) with $\nu_{g} \simeq 285 \mathrm{~cm}^{-1}$. However, a better description is

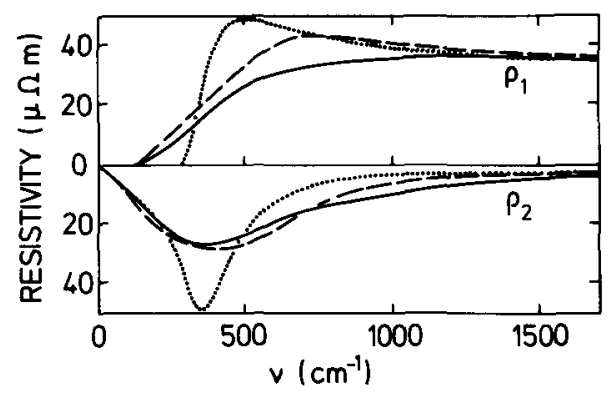

Fig. 3 Dynamical resistivities for low temperature; solid lines, experiment; dotted, theory with $2 \Delta / k T_{c}=4.6$; dashed, theory with a gap distribution (2.2 to 8 ).

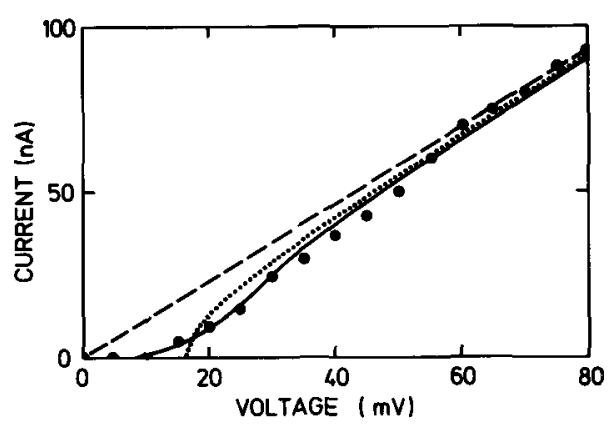

Fig. 4 Experimental characteristic (points); dotted, theory with $2 \Delta / k T_{c}=4.6$; solid, theory with a gap distribution ( 2.2 to 8 ); dashed, linear behavior.

obtained by assuming a gap distribution, that may be attributed to grains of slightly different electronic structure and to the anisotropy of the crystallites.

\section{TUNNEL EXPERIMENT}

We have also performed a tunnel experiment with a tungsten tip on the superconducting sample; the contact area was about $(1 \mu \mathrm{m})^{2}$. The current is small at small voltages across the contact and increases linearly at large voltages (Fig. 4). The characteristic cannot be described by a single energy gap, a distribution of gaps gives a more appropriate description; the distribution we used is the same as for the analysis of the far-infrared experiment. Our results are consistent with other tunnel experiments (8).

\section{CONCLUSION}

An energy gap $2 \Delta / k T_{c} \simeq 4.6$, or a distribution of gaps around this value, gives support for strong coupling superconductivity in $\mathrm{YBa}_{2} \mathrm{Cu}_{3} \mathrm{O}_{7}$. Both an influence of the superconductivity on a phonon mode and superconducting fluctuations are consistent with a two-dimensional character of the superconductivity.

\section{ACKNOWLEDGEMENTS}

We would like to thank L. Genzel for very stimulating discussions and to the Bruker Analytische Meßtechnik G.m.b.H. for making available an infrared Fourier spectrometer. The work was supported by the Bundesministerium für Forschung und Technologie.

\section{REFERENCES}

(1) G.A. Thomas et al., Phys.Rev.B 36 (1987) 846.

(2) D.A. Bonn et al., Phys.Rev.Lett. 58 (1987) 2249.

3) L. Genzel et al., Sol.St.Commun. 63 (1987) 843.

4) T.W. Noh et al., in print.

5 Z. Schlesinger et al., Phys. Rev.Lett. 59 (1987) 1958.

(6) W. Ose et al., Z.Phys. B, in print.

7) D.C. Mattis and J.Bardeen, Phys.Rev. 111 (1958) 412.

(8) J.R. Kirtley et al., Phys.Rev. B 35 (1987) 8846. 